\title{
Finite-dimensional description of nonlinear pulse propagation in optical-fiber couplers with applications to soliton switching
}

\author{
E. Caglioti \\ Dipartimento di Fisica, Università di Roma “La Sapienza," P. le Aldo Moro 7, 00185 Rome, Italy \\ S. Trillo and S. Wabnitz \\ Fondazione Ugo Bordoni, Via Baldassarre Castiglione 59, 00142 Roma, Italy \\ B. Crosignani and P. Di Porto \\ Dipartimento di Fisica, Università dell'Aquila, 67100 L'Aquila, Italy
}

Received May 14, 1989; accepted October 6, 1989

\begin{abstract}
We have developed a general formalism able to describe nonlinear pulse propagation in multimode optical fibers or waveguide couplers by means of a finite number of parameters, which are functions of the traveled distance. These parameters possess a direct physical meaning and can be interpreted, for a wide class of time-dependent interaction phenomena, as conjugate variables of a suitable Hamiltonian. The accuracy and the importance of the method are discussed with reference to a specific example of bimodal optical propagation, involving soliton switching and soliton instabilities.
\end{abstract}

\section{INTRODUCTION}

A wide class of nonlinear fiber propagation problems associated with the optical Kerr effect can be described in terms of a system of partial differential equations (PDE's), which represent the space-time evolution of the complex amplitudes of a modal expansion of the electric field. ${ }^{1}$ It is well established that, under approximations that are reasonable for picosecond pulses, this system takes the form of a set of, say, $N$ coupled nonlinear Schrödinger (NLS) equations. ${ }^{1}$ Unfortunately, only a few analytical solutions of such systems of equations are known, which usually take the form of temporally localized solitary wave packets. In the particular case of propagation in a monomode fiber, a single NLS equation results..$^{2-8}$ As is well known, this equation is exactly integrable both in the anomalous ${ }^{4}$ and in the normal -5 dispersion regimes by means of the inverse scattering transform (IST) method, which reveals the existence of a family of bright ${ }^{4,6}$ and dark ${ }^{5,7}$ solitons, respectively. NLS bright and dark solitons have been observed experimentally in the propagation along an optical fiber., 90 On the other hand, when a system of incoherently coupled (i.e., with coupling terms that are linearly proportional to the intensities of the components) NLS equations are considered, only a few special cases (with $N=2$ ) are completely integrable by means of the IST, ${ }^{11,12}$ whereas no such IST solutions have been found when linear coupling terms are present. Solitary wave solutions to systems of coupled NLS equations may be found, without resorting to the IST method, by means of direct methods. ${ }^{13-18}$ These solutions include polarization-modu- lated vector solitary waves in birefringent fibers ${ }^{16}$ and coupled dark and bright solitary waves that may be sustained either by cross-phase modulation ${ }^{17}$ or by polarization coupling due to fiber birefringence. ${ }^{18}$

If, on the one hand, serious difficulties are faced in trying to obtain analytical solutions to initial value problems involving a set of coupled NLS equations, on the other hand, there is a strong interest, in various applicative problems, in finding ways of characterizing the nonlinear evolution of pulses that propagate in mode-coupling structures. To this purpose, a popular approach has been that of forgoing an exact solution of the system of PDE's in favor of an approximate description of the pulse evolution based on a limited number of parameters of direct physical interpretation that obey a set of ordinary differential equations (ODE's). Since the early work of Kaup and Newell, ${ }^{19}$ a number of papers have appeared in which the particlelike nature of solitons has been exploited in order to derive, from conservation laws, ODE's for the adiabatic variation of some soliton parameters during the propagation. ${ }^{20-24}$ In particular, Refs. 20-22 have shown that, from the variational principle based on the field Lagrangian, one may obtain a set of ODE's for a given number of relatively slowly varying parameters, which determine the field amplitudes through a prescribed functional dependence. This method gives results that are generally in good agreement with those obtainable by means of analogous perturbative approaches based on the longitudinal variation of scattering parameters associated with the IST representation of the pulse. ${ }^{22,23}$ Alternatively, one may also prove, by direct insertion of the modal amplitudes into 
the set of NLS equations, that a suitable choice of soliton parameters yields equations for conjugate variables of a finite-dimensional Hamiltonian dynamical system. ${ }^{24}$

Finite-dimensional approaches to nonlinear wave propagation are particularly attractive in that one may gain an immediate global physical insight, for example, by means of drawing phase-space portraits or by finding motion invariants, into the dynamics of a solitonlike wave packet. For example, a description based on a Hamiltonian system with only a few degrees of freedom may permit an analytical determination, by means of well-developed techniques, ${ }^{25}$ of the regions in parameter space that may be subject to solitary wave instabilities, thus avoiding extensive and blind numerical searches (for example, with the beam-propagation method ${ }^{26}$ ). Finally, replacing a system of PDE's with a few coupled first-order ODE's is generally convenient also from a purely computational point of view, even though this may lead to reduced numerical accuracy.

In the present paper we present a systematic approach that permits the derivation, starting directly from a quite general class of field Lagrangians, of a self-consistent system of Hamilton equations for a finite set of suitably chosen parameters that characterize the interacting components of the pulse envelope. In this way we are able to generalize the results that were obtained in Ref. 24 for a specific propagation problem, where the resulting Hamiltonian form of the parameter ODE's looked somewhat fortuitous. The actual number of parameters is arbitrary, and clearly the accuracy of the finite-dimensional representation may be improved by increasing the number of parameters. These parameters possess an immediate physical meaning and give rise to a particularly simple and elegant finite-dimensional description.

The general formalism is applied in this paper to nonlinear pulse propagation, in the regime of anomalous chromatic dispersion, through two-mode coupling structures. Such nonlinear couplers have been of interest in recent years for demonstrating the potential of glass fibers for ultrafast alloptical switching. ${ }^{27}$ The two coupled modal amplitudes may represent, for example, the individual (or array) modes of a twin-core fiber, ${ }^{28-31}$ the two counterrotating circular polarization components of a linearly birefringent fiber, ${ }^{32,33}$ the unperturbed linear polarization eigenmodes of a periodically twisted fiber, ${ }^{34}$ or the two lowest-order modes of a single-core fiber or waveguide, with $^{35}$ or without ${ }^{36}$ gratingassisted coupling. Besides being of potential for these applications, coupling processes in two-mode structures are known to exhibit intriguing nonlinear dynamical properties even in the steady-state limit. ${ }^{31-33}$ As we shall show, the finite-dimensional perturbative approach makes it possible to appreciate more clearly what the effects are of the extra degrees of freedom that are provided by linear dispersion on the spatial instabilities and the self-switching of short optical pulses.

The practical interest in using two-mode fiber coupling structures in the anomalous-dispersion regime and with pulses whose values of peak power and width are sufficiently close to the values required for the formation of solitons is the possibility of avoiding the breakup of the output pulse profile, which typically occurs in the quasi-continuous-wave regime. ${ }^{37-40}$ This phenomenon is detrimental if, for exam- ple, one wants to cascade different switching elements and is due to the fact that different portions of a sufficiently long input pulse couple with different efficiencies and coupling periods, according to their own instantaneous power level. A substantial improvement in the switching characteristics may result when square pulses are used, ${ }^{41}$ although the synthesis of such pulses with picosecond durations requires considerable experimental effort. ${ }^{42}$ On the other hand, it has been pointed out and recently experimentally demonstrated that the particlelike behavior of soliton pulses may inhibit pulse breakup in different examples of ultrafast alloptical switching devices. ${ }^{43-47}$ Moreover, in the specific case of interest here, namely, the interaction between two linearly and nonlinearly coupled modes, the existence of soliton instabilities has been numerically and analytically investigated by several authors. ${ }^{45,47-50}$

The paper is organized as follows: in Section 2 we present specific physical examples of two-mode fiber couplers for which the time-dependent propagation is described by systems of NLS equations. In Section 3 we derive a finitedimensional representation of the propagation of a pulse. From a field Lagrangian that is written in a general form, one may reduce the Lagrange equations to a system of coupled first-order ODE's for a set of suitable time-independent Hamiltonian conjugate variables. Finally, in Section 4 we discuss an example in which the method is applied to investigating soliton switching and instability phenomena in a nonlinear directional coupler.

\section{COUPLED-MODE EQUATIONS}

Here we give a brief résumé of the coupled-mode equations that govern the propagation of two coupled pulses in different types of fiber couplers. These include the cases of a linearly birefringent fiber, a periodically twisted birefringent fiber, and a single-polarization, dual-core fiber. Along with the coupled-mode equations, the respective field Lagrangians are also listed below.

\section{A. Birefringent Fiber}

The electromagnetic field propagating in a linearly birefringent optical fiber along the positive direction of the $z$ axis, which is aligned with its symmetry axis, can be expressed as a superposition of linearly polarized guided modes, with mean optical frequency $\omega_{0}$, in the form

$$
\begin{aligned}
\mathbf{E}(x, y, z, t)= & {\left[\mathbf{x} E_{x}(z, t) \exp \left(i \beta_{x} z\right)+\mathbf{y} E_{y}(z, t) \exp \left(i \beta_{y} z\right)\right] } \\
& \times f(x, y) \exp \left(-i \omega_{0} \mathbf{t}\right),
\end{aligned}
$$

where $\beta_{x, y}\left(\omega_{0}\right)$ are their propagation constants and $f(x, y)$ is a modal transverse configuration (which is assumed equal for the two modes, which is reasonable in a weakly birefringent fiber). The slowly varying amplitudes $A_{x}(z, t)=E_{x}(z$, $t) \exp \left[i\left(\beta_{x}-\beta_{y}\right) z / 2\right]$ and $A_{y}(z, t)=E_{y}(z, t) \exp \left[-i\left(\beta_{x}-\beta_{y}\right) z /\right.$ 2] obey the set of PDE's ${ }^{45,15,16,51}$

$$
\begin{aligned}
i\left(\frac{\partial A_{x}}{\partial z}+\frac{1}{V_{x}} \frac{\partial A_{x}}{\partial t}\right)-\frac{\alpha_{x}}{2} \frac{\partial^{2} A_{x}}{\partial t^{2}}+\frac{\Delta \beta}{2} A_{x} \\
+R\left[\left|A_{x}\right|^{2}+(1-B)\left|A_{y}\right|^{2}\right] A_{x}+R B A_{y}{ }^{2} A_{x}{ }^{*}=0
\end{aligned}
$$




$$
\begin{aligned}
i\left(\frac{\partial A_{y}}{\partial z}+\right. & \left.\frac{1}{V_{y}} \frac{\partial A_{y}}{\partial t}\right)-\frac{\alpha_{y}}{2} \frac{\partial^{2} A_{y}}{\partial t^{2}}-\frac{\Delta \beta}{2} A_{y} \\
& +R\left[\left|A_{y}\right|^{2}+(1-B)\left|A_{x}\right|^{2}\right] A_{y}+R B A_{x}{ }^{2} A_{y}{ }^{*}=0
\end{aligned}
$$

where $V_{x, y}=\left(\mathrm{d} \beta_{x, y} /\left.\mathrm{d} \omega\right|_{\omega 0}\right)^{-1}$ are the group velocities of the modes, $\alpha_{x, y}=\mathrm{d}^{2} \beta_{x, y} /\left.\mathrm{d} \omega^{2}\right|_{\omega_{0}}$ represent chromatic dispersion, and $\Delta \beta=\beta_{x}\left(\omega_{0}\right)-\beta_{y}\left(\omega_{0}\right)$ is the fiber birefringence. Moreover, $R=\omega_{0} n_{2 I} / c A$, where $A$ is the effective common area of the modes and $n_{2 I}$ is the nonlinear refractive-index coefficient (the total refractive index is $n=n_{0}+n_{2 I} I, I$ being the intensity). We assumed in Eqs. (2) that the modes are mixed by the isotropic nonlinear vectorial displacement ${ }^{52}$

$$
\mathbf{D}_{\mathrm{NL}}=\epsilon_{0} \chi\left[(1-B)\left(\mathbf{E} \cdot \mathbf{E}^{*}\right) \mathbf{E}+B(\mathbf{E} \cdot \mathbf{E}) \mathbf{E}^{*}\right]
$$

where $B=\chi_{x y y x} / \chi_{x x x x}$ and $\chi=3 \chi_{x x x x}$ (note that $0<B<1$ and $B=1 / 3$ for silica).

The nonlinear Eqs. (2) for the fields may be expressed in terms of a variational formulation by means of a Lagrangian density $\mathcal{L}$. This is a functional of the modal amplitudes, of their partial derivatives with respect to $z$ and $t$, and of the complex conjugates of these quantities (which are regarded as independent variables). The Lagrangian density that generates Eqs. (2) is

$$
\begin{aligned}
\mathcal{L}= & -\operatorname{Im}\left(A_{x}^{*} \frac{\partial A_{x}}{\partial z}+A_{y}^{*} \frac{\partial A_{y}}{\partial z}\right) \\
& -\operatorname{Im}\left(\frac{1}{V_{x}} A_{x} * \frac{\partial A_{x}}{\partial t}+\frac{1}{V_{y}} A_{y} * \frac{\partial A_{y}}{\partial t}\right) \\
& +\frac{\alpha_{x}}{2} \frac{\partial A_{x}^{*}}{\partial t} \frac{\partial A_{x}}{\partial t}+\frac{\alpha_{y}}{2} \frac{\partial A_{y}^{*}}{\partial t} \frac{\partial A_{y}}{\partial t} \\
& +\frac{\Delta \beta}{2}\left(\left|A_{x}\right|^{2}-\left|A_{y}\right|^{2}\right)+\frac{R B}{2}\left(\left|A_{x}\right|^{4}+\left|A_{y}\right|^{4}\right) \\
& +\frac{R}{2}(1-B)\left(\left|A_{x}\right|^{2}+\left|A_{y}\right|^{2}\right)^{2}+\frac{R B}{2}\left(A_{y}{ }^{2} A_{x}{ }^{* 2}+A_{y}{ }^{* 2} A_{x}{ }^{2}\right) .
\end{aligned}
$$

The set of equations describing the evolution of the modal amplitudes $A_{x, y}$ can be obtained from the Lagrangian density $\mathcal{L}=\mathcal{L}\left[\left\{A_{j}\right\},\left\{A_{j z}\right\},\left\{A_{j t}\right\},\left\{A_{j}{ }^{*}\right\},\left\{A_{j z}{ }^{*}\right\},\left\{A_{j t}{ }^{*}\right\}\right]$, with $j=x, y$, by the variational principle

$$
\delta \iint \mathrm{d} z \mathrm{~d} t \mathcal{L}=0,
$$

which in turn implies the Lagrange equations

$$
\begin{gathered}
\frac{\partial}{\partial t} \frac{\partial \mathcal{L}}{\partial A_{j t}}+\frac{\partial}{\partial z} \frac{\partial \mathcal{L}}{\partial A_{j z}}-\frac{\partial \mathcal{L}}{\partial A_{j}}=0, \\
\frac{\partial}{\partial t} \frac{\partial \mathcal{L}}{\partial A_{j t}{ }^{*}}+\frac{\partial}{\partial z} \frac{\partial \mathcal{L}}{\partial A_{j z}{ }^{*}}-\frac{\partial \mathcal{L}}{\partial A_{j}{ }^{*}}=0, \quad j=x, y,
\end{gathered}
$$

where $A_{j t} \equiv \partial A_{j} / \partial t$ and $A_{j z} \equiv \partial A_{j} / \partial z$. The first and the second of Eqs. (6) yield the coupled-mode Eqs. (2) and their complex-conjugate equations, respectively.

Let us now rewrite Eqs. (2) in terms of circularly polarized modes, which are given by the relations ${ }^{63}$

$$
\begin{aligned}
& A_{+}=\frac{1}{\sqrt{2}}\left(A_{x}+i A_{y}\right), \\
& A_{-}=\frac{1}{\sqrt{2}}\left(A_{x}-i A_{y}\right) .
\end{aligned}
$$

From Eqs. (2) one obtains

$$
\begin{aligned}
& \begin{aligned}
i\left(\frac{\partial A_{+}}{\partial z}+\frac{1}{\delta V} \frac{\partial A_{-}}{\partial s}\right) & -\frac{\alpha}{2} \frac{\partial^{2} A_{+}}{\partial s^{2}}+k A_{-} \\
& +R\left[(1-B)\left|A_{+}\right|^{2}+(1+B)\left|A_{-}\right|^{2}\right] A_{+}=0, \\
i\left(\frac{\partial A_{-}}{\partial z}+\frac{1}{\delta V} \frac{\partial A_{+}}{\partial s}\right)- & \frac{\alpha}{2} \frac{\partial^{2} A_{-}}{\partial s^{2}}+k A_{+} \\
+ & R\left[(1-B)\left|A_{-}\right|^{2}+(1+B)\left|A_{+}\right|^{2}\right] A_{-}=0,
\end{aligned}
\end{aligned}
$$

where $k \equiv \Delta \beta / 2$. In Eqs. (8) we assumed equal dispersion coefficients for the linear polarization modes $\alpha_{x}=\alpha y \equiv \alpha$, and we used the delayed time variables $s=t-z / V_{g}$, where $V_{g}{ }^{-1} \equiv$ $\left(V_{x}^{-1}+V_{y}^{-1}\right) / 2$ and $\delta V^{-1} \equiv\left(V_{x}^{-1}-V_{y}{ }^{-1}\right) / 2$ are the inverse of the average group velocity and of the group-velocity difference, respectively. The Lagrangian density that generates Eqs. (8) is

$$
\begin{aligned}
\mathcal{L}= & -\operatorname{Im}\left(A_{-} * \frac{\partial A_{-}}{\partial z}+A_{+} * \frac{\partial A_{+}}{\partial z}\right) \\
& -\frac{1}{\delta V} \operatorname{Im}\left(A_{+} * \frac{\partial A_{-}}{\partial s}+A_{-} * \frac{\partial A_{+}}{\partial s}\right) \\
& +\frac{\alpha}{2}\left(\frac{\partial A_{+}{ }^{*}}{\partial s} \frac{\partial A_{+}}{\partial s}+\frac{\partial A_{-} *}{\partial s} \frac{\partial A_{-}}{\partial s}\right)+k\left(A_{+}{ }^{*} A_{-}+A_{-}{ }^{*} A_{+}\right) \\
& +\frac{R(1-B)}{2}\left(\left|A_{+}\right|^{4}+\left|A_{-}\right|^{4}\right)+R(1+B)\left|A_{+}\right|^{2}\left|A_{-}\right|^{2} \text { (9) }
\end{aligned}
$$

In Eqs. (8) and (9) the coefficient $k$, which is due to fiber birefringence, leads at low powers to complete periodic power exchange between the two circularly polarized components. The spatial period of the coupling is the beat length $L_{b}=2 L_{c}=\pi / k$, where $L_{c}$ is the coupling length. In the limit $k=0$, the circularly polarized waves experience just a nonlinear phase shift (this leads to a uniform rotation of the polarization ellipse ${ }^{52,53}$ ), which is due to the self- and crossphase modulation terms [with coefficients $R(1-B)$ and $R(1$ $+B$ ), respectively]. Both in the steady state and in the dispersive regime, at relatively high peak powers the presence of both linear coupling and nonlinear phase shifting leads to polarization-instability phenomena. ${ }^{32,33,45-50}$

\section{B. Periodically Twisted Fiber}

Consider now the case of a birefringent fiber, in which longitudinally periodic coupling between the two linear polarizations is introduced by periodic twisting. In the regime of purely linear coupling, this fiber may be used for wavelength-dependent polarization rotation. When the fiber is placed between two crossed polarizers, a bandpass filter results, with typical bandwidths of a few nanometers. ${ }^{34}$ Whenever the total field is written as the superposition of linearly polarized modes [see Eq. (1)], the slowly varying envelope amplitudes $A_{x, y}$ that were introduced in Subsection 2.A obey the equations 


$$
\begin{gathered}
i\left(\frac{\partial A_{x}}{\partial z}+\frac{1}{V_{x}} \frac{\partial A_{x}}{\partial t}\right)-\frac{\alpha_{x}}{2} \frac{\partial^{2} A_{x}}{\partial t^{2}}+\frac{\Delta \beta}{2} A_{x}+2 k \cos \left(\beta_{0} z+\phi\right) A_{y} \\
+R\left[\left|A_{x}\right|^{2}+(1-B)\left|A_{y}\right|^{2}\right] A_{x}+R B A_{y}{ }^{2} A_{x}{ }^{*}=0
\end{gathered}
$$

With respect to Eqs. (2), note the presence here of a new term, which is due to periodic coupling between the two linearly polarized modes. This leads, at low powers, to complete rotation of the polarization state of a linearly polarized beam, which is initially aligned with the principal birefringence axes. ${ }^{34}$ Moreover, the group-velocity mismatch between the two coupled linear polarization modes is responsible for the dispersive properties of the coupling structure that are inherent in the filtering action. ${ }^{54}$

Equations (10) may be simplified by introducing the slowly varying amplitudes

$$
a_{x, y} \equiv E_{x, y} \exp \left[ \pm \frac{i}{2}(\delta z-\phi)\right]
$$

where the coefficient $\delta \equiv\left\{\left[\beta_{x}\left(\omega_{0}\right)-\beta_{y}\left(\omega_{0}\right)\right]-\beta_{0}\right\} / 2$ is the detuning from resonance [where $\Delta \beta\left(\omega_{0}\right)=\beta_{0}$ ]. Neglecting the terms that correspond to fast spatial oscillations (rotating-wave approximation), from Eqs. (10) one obtains

$$
\begin{aligned}
& i\left(\frac{\partial a_{x}}{\partial z}+\frac{1}{\delta V} \frac{\partial a_{x}}{\partial s}\right)-\frac{\alpha}{2} \frac{\partial^{2} a_{x}}{\partial s^{2}}+\delta a_{x}+k a_{y} \\
& +R\left[\left|a_{x}\right|^{2}+(1-B)\left|a_{y}\right|^{2}\right] a_{x}=0, \\
& i\left(\frac{\partial a_{x}}{\partial z}-\frac{1}{\delta V} \frac{\partial a_{y}}{\partial s}\right)-\frac{\alpha}{2} \frac{\partial^{2} a_{y}}{\partial s^{2}}-\delta a_{y}+k a_{x} \\
& +R\left[\left|a_{y}\right|^{2}+(1-B)\left|a_{x}\right|^{2}\right] a_{y}=0,
\end{aligned}
$$

where $s$ and $\delta V$ are the delayed time and the group-velocity difference, respectively, as they were defined in Subsection 2.A. The field Lagrangian density that corresponds to Eqs. (12) is

$$
\begin{aligned}
\mathcal{L}= & -\operatorname{Im}\left(a_{x} * \frac{\partial a_{x}}{\partial z}+a_{y} * \frac{\partial a_{y}}{\partial z}\right) \\
& -\operatorname{Im}\left(\frac{1}{\delta V} a_{x}^{*} \frac{\partial a_{x}}{\partial s}-\frac{1}{\delta V} a_{y}{ }^{*} \frac{\partial a_{y}}{\partial s}\right) \\
& +\frac{\alpha}{2}\left(\frac{\partial a_{x}{ }^{*}}{\partial s} \frac{\partial a_{x}}{\partial s}+\frac{\partial a_{y}{ }^{*}}{\partial s} \frac{\partial a_{y}}{\partial s}\right) \\
& +k\left(a_{x} a_{y}{ }^{*}+a_{y} a_{x}{ }^{*}\right)+\delta\left(\left|a_{x}\right|^{2}-\left|a_{y}\right|^{2}\right) \\
& +\frac{R B}{2}\left(\left|a_{x}\right|^{4}+\left|a_{y}\right|^{4}\right)+\frac{R}{2}(1-B)\left(\left|a_{x}\right|^{2}+\left|a_{y}\right|^{2}\right)^{2} .
\end{aligned}
$$

It is worth noting that Eqs. (12) and (13) are a correct representation of the propagation in the nonlinear rocking rotator fiber filter only for relatively low peak power levels. These are such that the nonlinear power exchange that occurs between the modes over a distance of the order of the birefringence beat length $L_{b}$ may be neglected. At higher power levels, when the nonlinear power exchange may no longer be neglected, the rotating-wave approximation does not apply. In this case, even in the stationary regime the nonautonomous coupled-mode equations do not possess the integrability property: spatial Hamiltonian chaos in the evolution of the polarization may occur. ${ }^{55,56}$

\section{Dual-Core Fiber}

The scalar field that travels in a weakly coupled dual-core isotropic fiber may be expressed as the superposition of the modes of the two individual cores:

$$
\begin{aligned}
E(x, y, z, t)= & {\left[A_{1}(z, t) f_{1}(x, y) \exp \left(i \beta_{1} z\right)\right.} \\
& \left.+A_{2}(z, t) f_{2}(x, y) \exp \left(i \beta_{2} z\right)\right] \exp \left(i \omega_{0} t\right) .
\end{aligned}
$$

For identical cores, the slowly varying modal amplitudes $A_{1,2}$ obey the system of coupled PDE's ${ }^{46}$

$$
\begin{aligned}
& \left(i \frac{\partial}{\partial z}-\frac{\alpha}{2} \frac{\partial^{2}}{\partial s^{2}}\right) A_{1}+k A_{2}+R\left|A_{1}\right|^{2} A_{1}=0, \\
& \left(i \frac{\partial}{\partial z}-\frac{\alpha}{2} \frac{\partial^{2}}{\partial s^{2}}\right) A_{2}+k A_{1}+R\left|A_{2}\right|^{2} A_{2}=0,
\end{aligned}
$$

where $s$ is the delayed time in a reference frame that travels with the common group velocity $V_{g}$. Equations (15) are adequate to describe situations in which the isotropic part of the nonlinearity and the linear coupling represent weak perturbations to the ideally uncoupled propagation of the modes of the two identical cores. The Lagrangian density that corresponds to Eqs. (15) is

$$
\begin{aligned}
\mathcal{L}= & -\operatorname{Im}\left(A_{1}{ }^{*} \frac{\partial A_{2}}{\partial z}+A_{2}^{*} \frac{\partial A_{1}}{\partial z}\right) \\
& +\frac{\alpha}{2}\left(\frac{\partial A_{1}^{*}}{\partial s} \frac{\partial A_{1}}{\partial s}+\frac{\partial A_{2}{ }^{*}}{\partial s} \frac{\partial A_{2}}{\partial s}\right) \\
& +k\left(A_{1}^{*} A_{2}+A_{2}^{*} A_{1}\right)+\frac{R}{2}\left(\left|A_{1}\right|^{4}+\left|A_{2}\right|^{4}\right) .
\end{aligned}
$$

As one can see by inspecting Eqs. (9), (13), and (16), there is a close similarity between the field Lagrangians that describe the interaction of two circularly polarized modes in a birefringent fiber, of linearly polarized modes in a periodically twisted fiber, and of scalar individual core modes of a twin-core-fiber nonlinear directional coupler.

\section{HAMILTONIAN FORMALISM}

We deal here with a quite general form of field Lagrangians, which are associated with the nonlinear interaction between two guided modes. Extension to the general case of $N$ coupled modes is rather straightforward and will not be discussed here. The treatment that follows may be immediately applied to deal with any one of the cases that appear in Section 2. We show how two coupled NLS equations may be reduced to a Hamiltonian system of ODE's for a certain set of $z$-dependent parameters. We consider Lagrangian densities that take the general form 


$$
\begin{aligned}
\mathcal{L}= & \sum_{j=1}^{2}\left\{\frac { i } { 2 } \left[\left(u_{j}^{*} \frac{\partial u_{j}}{\partial z}-u_{j} \frac{\partial u_{j}^{*}}{\partial z}\right)-(-1)^{j} \frac{m}{V}\left(u_{j}^{*} \frac{\partial u_{j}}{\partial t}\right.\right.\right. \\
& \left.\left.\left.-u_{j} \frac{\partial u_{j}^{*}}{\partial t}\right)+\frac{n}{V}\left(u_{j}^{*} \frac{\partial u_{3-j}}{\partial t}-u_{j} \frac{\partial u_{3-j}}{\partial t}\right)\right]\right\} \\
& +-\sum_{j=1}^{2}\left( \pm \frac{1}{2}\left|\frac{\partial u_{j}}{\partial t}\right|^{2}-\frac{1}{2}\left|u_{j}\right|^{4}\right)+\Delta\left(\left|u_{1}\right|^{2}-\left|u_{2}\right|^{2}\right) \\
& +\kappa\left(u_{1} u_{2}^{*}+u_{1}^{*} u_{2}\right)+\gamma\left|u_{1}\right|^{2}\left|u_{2}\right|^{2}+\rho\left(u_{2}{ }^{2} u_{1}^{* 2}+u_{2}^{* 2} u_{1}{ }^{2}\right),
\end{aligned}
$$

where $m$ and $n$ can independently assume the values of 0 and 1 and the upper (lower) sign in front of the dispersive term holds in case of propagation in the anomalous- (normal-) dispersion regime. The explicit form of the coupled-mode equations that are satisfied by the modal envelopes $\left(u_{1}, u_{2}\right)$ may be obtained by writing the Lagrange equations [see Eqs. (6)] that correspond to Eq. (17). They read as

$$
\begin{aligned}
& i\left(\frac{\partial u_{1}}{\partial z}+\frac{m}{V} \frac{\partial u_{1}}{\partial t}+\frac{n}{V} \frac{\partial u_{2}}{\partial t}\right) \pm \frac{1}{2} \frac{\partial^{2} u_{1}}{\partial t^{2}} \\
& +\Delta u_{1}+\kappa u_{2}+\left(\left|u_{1}\right|^{2}+\gamma\left|u_{2}\right|^{2}\right) u_{1}+\rho u_{2}^{2} u_{1}^{*}=0, \\
& i\left(\frac{\partial u_{2}}{\partial z}-\frac{m}{V} \frac{\partial u_{2}}{\partial t}+\frac{n}{V} \frac{\partial u_{1}}{\partial t}\right) \pm \frac{1}{2} \frac{\partial^{2} u_{2}}{\partial t^{2}} \\
& -\Delta u_{2}+\kappa u_{1}+\left(\left|u_{2}\right|^{2}+\gamma\left|u_{1}\right|^{2}\right) u_{2}+\rho u_{1}^{2} u_{2}^{*}=0 \text {. }
\end{aligned}
$$

Equations (17) and (18) appear in a normalized form: we have adopted the usual dimensionless soliton units, where $z$ is expressed in terms of a characteristic length $z_{c} \equiv t_{s}{ }^{2} /|\alpha|\left(z_{0}\right.$ $=\pi z_{c} / 2$ is the period of higher-order solitons of a single NLS equation), ${ }^{9} t$ is the retarded time, which is in units of the temporal width $t_{s}$ of the fundamental soliton solution of a single NLS equation $[u=\operatorname{sech}(t)]$, and the field amplitudes are normalized accordingly. ${ }^{9,45-51}$ We have also introduced a normalized group-velocity difference $V=\left.\delta V\right|_{\alpha} \mid / t_{s}$. The mismatch term $\Delta$ between the propagation constants of the two guides may be due, for example, to the presence of uniform twisting or optical activity in a birefringent fiber (Subsection 2.A) or to core asymmetries in a dual-core fiber (Subsection 2.B).

Clearly, Eqs. (17) and (18) include as particular cases all the equations discussed in Section 2. For example, the linearly polarized modes of a birefringent silica (i.e., for $B=$ 1/3) fiber satisfy Eqs. (18) with $m=1, n=0, \Delta=\Delta \beta t_{s}^{2} / 2|\alpha|, \kappa$ $=0, \gamma=1-B=2 / 3$, and $\rho=B=1 / 3$. The interaction of circular modes in the birefringent fiber is described by Eqs. (18) with $n=1, m=0, \Delta=0, \gamma=(1+B) /(1-B)=2, \rho=0$, and $\kappa \equiv k t_{s}^{2} /|\alpha|=\Delta \beta t_{s}^{2} / 2|\alpha|=z_{0} / L_{c}=P_{c}|1-\gamma| / 4 P_{s}$, where $P_{c}$ and $P_{s}$ (in watts) are the continuous-wave critical switching power and the fundamental soliton power of a single NLS equation, respectively. In the case of linearly polarized modes, which are coupled along a rocking rotator glass-fiber filter, Eqs. (18) apply with $m=1, n=0, \Delta=\delta t_{s}{ }^{2} /|\alpha|, \kappa=k t_{s}^{2} /$ $|\alpha|=P_{c} /\left(12 P_{s}\right), \gamma=(1-B)=2 / 3$, and $\rho=0$. Finally, Eqs. (18) hold for a dual-core fiber with $m=n=0, \Delta=0, k=k t_{s}^{2} /$ $|\alpha|$, and $\gamma=\rho=0$. Equations (17) and (18) may also describe other types of interaction: For example, consider the coupling between two lowest-order modes in a multimode fiber $^{1,36}$ or between two pulses with identical polarization and slightly different average frequency that travel in the same mode of an isotropic fiber. ${ }^{17}$

Let us introduce the Lagrangian $L$, which is defined as

$$
L=\int_{-\infty}^{+\infty} \mathcal{L} \mathrm{d} t
$$

so that the variational principle [Eq. (5)] may be expressed as

$$
\delta \int L \mathrm{~d} z=0
$$

First, let us suppose, in the spirit of the finite-dimensional approach, that we may characterize each mode amplitude

$$
u_{j}(z, t)=\left|u_{j}(z, t)\right| \exp \left[i \psi_{j}(z, t)\right] \quad(j=1,2)
$$

in terms of a finite number, say, $2 N+2$, of $z$-dependent parameters

$$
M_{n}^{(j)}(z), a_{n}{ }^{(j)}(z) \quad(n=0,1,2,3, \ldots, N) \quad(j=1,2),
$$

which are defined as follows:

$$
\begin{gathered}
M_{n}^{(j)}(z)=\int_{-\infty}^{+\infty} t^{n}\left|u_{j}(z, t)\right|^{2} \mathrm{~d} t, \\
\Psi_{j}(z, t)=\sum_{n=0}^{N} a_{n}{ }^{(j)}(z) t^{n} .
\end{gathered}
$$

Second, we assume that the $\left|u_{n}(z, t)\right|^{2}$ 's are of the form

$$
\left|u_{j}(z, t)\right|^{2}=F_{j}\left[M_{0}^{(j)}(z), M_{1}^{(j)}(z), \ldots, M_{N}^{(j)}(z), t\right],
$$

where $F_{j}$ is an arbitrary function that satisfies Eq. (23). We therefore look for the solution of the variational problem [Eq. (20)] with $u$ in the class of functions that is defined by Eq. (25). For example, when $N=2$, we may write

$$
\left|u_{j}(z, t)\right|^{2}=\frac{M_{0}^{(j)}}{\sigma_{j}} f_{j}\left(\frac{t-\tau_{j}}{\sigma_{j}}\right),
$$

where $\tau_{j}$ and $\sigma_{j}$ are obviously related to the first- and secondorder momenta $M_{0}^{(j)}$ and $M_{2}{ }^{(j)}$, respectively, and $f_{j}$ is an arbitrary normalized function with mean value and variance equal to 1. The accuracy of the approximation depends, of course, both on the choice of the number $N$ of degrees of freedom and on the form of the $f_{j}$ 's.

According to the above considerations, we have, in general, that

$$
L=L\left[a_{n}^{(j)}, \dot{a}_{n}{ }^{(j)}, M_{n}^{(j)}, \dot{M}_{n}^{(j)}\right]
$$

where the dots stand for derivation with respect to $z$. Set

$$
L \equiv L_{0}+L_{1} \text {, }
$$

where by definition we choose

$$
\begin{aligned}
L_{0} & \equiv \int_{-\infty}^{+\infty} \mathcal{L}_{0} \mathrm{~d} t=\int_{-\infty}^{+\infty} \sum_{j=1}^{2} \frac{i}{2}\left(u_{j}^{*} \frac{\partial u_{j}}{\partial z}-u_{j} \frac{\partial u_{j}^{*}}{\partial z}\right) \mathrm{d} t \\
& =-\sum_{j=1}^{2} \int_{-\infty}^{+\infty}\left|u_{j}\right|^{2} \frac{\partial \psi_{j}}{\partial z} \mathrm{~d} t=-\sum_{j=1}^{2} \sum_{n=0}^{N} \dot{a}_{n}{ }^{(j)} M_{n}{ }^{(j)}
\end{aligned}
$$




$$
L_{1} \equiv \int_{-\infty}^{+\infty}\left(\mathcal{L}-\mathcal{L}_{0}\right) \mathrm{d} t
$$

We may then rewrite the Lagrange equations associated with the variational principle [Eqs. (6): here the $A_{j}$ 's are replaced by the $u_{j}$ 's and $(j=x, y)$ with $\left.(j=1,2)\right]$. We obtain

$$
\begin{gathered}
\frac{\partial}{\partial z} \frac{\partial L}{\partial \dot{a}_{n}^{(j)}}-\frac{\partial L}{\partial a_{n}^{(j)}}=0, \\
\frac{\partial}{\partial z} \frac{\partial L}{\partial \dot{M}_{n}^{(j)}}-\partial L_{\partial M_{n}^{(j)}}=0 \\
(j=1,2), \quad(n=0,1,2,3, \ldots, N) .
\end{gathered}
$$

By inspecting Eqs. (24)-(26) and by exploiting the special form of the Lagrangian in Eq. (17), one may immediately verify that

$$
\frac{\partial L}{\partial \dot{a}_{n}^{(j)}}=\frac{\partial L_{0}}{\partial \dot{a}_{n}^{(j)}}=M_{n}^{(j)},
$$

so that the moments $M_{n}{ }^{(j)}$ represent the conjugate momenta of the variables $a_{n}^{(j)}$. Besides, the set of Eqs. (31) reduces to

$$
\begin{aligned}
& \frac{\partial}{\partial z} M_{n}^{(j)}=\frac{\partial L}{\partial a_{n}^{(j)}}=\frac{\partial L_{1}}{\partial a_{n}^{(j)}}, \\
& -\frac{\partial L}{\partial M_{n}^{(j)}}=-\frac{\partial L_{0}}{\partial M_{n}^{(j)}}-\frac{\partial L_{1}}{\partial M_{1}^{(j)}}=\dot{a}_{n}^{(j)}-\frac{\partial L_{1}}{\partial M_{n}^{(j)}}=0 .
\end{aligned}
$$

These are Hamiltonian equations of a dynamical system that is described by the set of conjugate variables $M_{n}{ }^{(j)}, a_{n}{ }^{(j)}$, whose Hamiltonian $H$ is given by $L_{1}$, that is,

$$
\begin{aligned}
& \frac{\mathrm{d} M_{n}^{(j)}}{\mathrm{d} z}=-\frac{\partial L_{1}}{\partial a_{n}{ }^{(j)}} \equiv-\frac{\partial H}{\partial a_{n}^{(j)}}, \\
& \frac{\mathrm{d}{a_{n}}^{(j)}}{\mathrm{d} z}=\frac{\partial L_{1}}{\partial M_{n}^{(j)}} \equiv \frac{\partial H}{\partial M_{n}^{(j)}} .
\end{aligned}
$$

The Hamiltonian $H$ is obviously also a conserved quantity of the original PDE's [Eqs. (18)]. Note that the reduction procedure presented above is by no means limited to the specific Lagrangian in Eq. (17): rather, the method is valid in all cases when the term $L_{1}$ does not exhibit an explicit dependence on both $\partial M_{n}^{(j)} / \partial z$ and $a_{n}^{(j)}$. Therefore the method may be applied with great generality to different physical situations, also beyond the framework of nonlinear optics. Note also that it is the particular choice of the parameters $M_{n}{ }^{(j)}$ and $a_{n}{ }^{(j)}$ in Eqs. (23) and (24) that permitted the reduction by a factor of 2 (from $2 N+2$ to $N+1$ ) of the number of degrees of freedom of the problem. This is a consequence of the peculiar form assumed by the kinetic part $L_{0}$, which in turn implies that $\partial L_{0} / \partial a_{n}{ }^{(j)}=\partial L_{0} / \partial\left[\partial M_{n}{ }^{(j)} /\right.$ $\partial z]=0$. By using the above formalism one may recover in a much simpler way the results of Ref. 24, where the Hamiltonian form of the parameter equations was obtained through a constructive and rather cumbersome approach. This consisted in explicitly deriving the $z$ evolution of $M_{0}^{(j)}, M_{1}{ }^{(j)}$, $M_{2}{ }^{(j)}$, and $a_{0}^{(j)}, a_{1}{ }^{(j)}, a_{2}{ }^{(j)}$ by direct insertion into the set of PDE's. In order to obtain the Hamiltonian $H$ in explicit form one must perform the integrations in Eq. (30). Even in the simple case with $N=2$ and with Gaussian pulse shapes this may lead to rather lengthy expressions: an example of such calculation is given in Appendix A.

\section{SOLITON SWITCHING AND INSTABILITIES}

The effectiveness and accuracy of the finite-dimensional characterization of pulse propagation in coupling structures are investigated here in some specific cases involving nonlinear directional couplers or birefringent fibers. In particular, we are interested in soliton switching and instability phenomena, so that the analysis is restricted to propagation in the anomalous-dispersion regime. The method requires specification both of the number $2(N+1)$ of momenta and of the particular form of the functions $F_{i}$ appearing in Eq. (25). Numerical simulations ${ }^{46}$ have indicated that under certain conditions solitonlike input pulses may periodically couple between the two modes of the coupler without appreciable changes in their shapes, so that we may choose $N=2$. In addition, the switching properties and the soliton instability phenomena do not to critically depend on the temporal shaping of the input pulses, which justifies the choice of Gaussian pulse profiles. This choice corresponds to the example reported in Appendix A and leads to substantially simpler expressions for the Hamiltonian with respect to the hyperbolic secant case. The interaction of weakly coupled modes may be described by Eqs. (8) or (15), so we shall set $\rho$ $=0$ and $\Delta=0$. We are essentially interested here in studying propagation over relatively short interaction lengths (of the order of the linear beat length), so we may also neglect any group-velocity difference. Therefore, according to Eq. (30), the Hamiltonian is $H=L_{1} \equiv H_{1}+H_{2}+H_{\text {int }}$, where

$$
\begin{gathered}
H_{j}=\frac{1}{2} \int_{-\infty}^{+\infty}\left(-\left|\frac{\partial u_{j}}{\partial t}\right|^{2}+\left|u_{j}\right|^{4}\right) \mathrm{d} t \quad(j=1,2), \\
H_{\text {int }}=\int_{-\infty}^{+\infty}\left(\gamma\left|u_{1}\right|^{2}\left|u_{2}\right|^{2}+2 \kappa \operatorname{Re}\left\{u_{1} u_{2}^{*}\right\}\right) \mathrm{d} t .
\end{gathered}
$$

We consider here, for the sake of simplicity, the case of initially unmodulated pulses. Since we have also neglected the presence of group-velocity difference $V$, one obtains $M_{1}^{(j)}(z)=a_{1}^{(j)}(z)=0, j=1,2$. As a consequence, the evolution of each pulse may be characterized by four parameters only (instead of six), and propagation described by the pair of NLS Eqs. (18) is studied in terms of a three-degreesof-freedom Hamiltonian system of ODE's. As is shown below, the Hamiltonian in Eqs. (35) is considerably simpler than the general case in Eq. (A3) below. The total Hamiltonian $H=H_{1}+H_{2}+H_{\text {int }}$ may be expressed as a function of the eight momenta $a_{0}{ }^{(j)}, a_{2}^{(j)}, M_{0}{ }^{(j)}, M_{2}{ }^{(j)}(j=1,2)$ and takes the form

$$
\begin{aligned}
& H_{j}=-\left\{\frac{1}{8} \frac{\left[M_{0}^{(j)}\right]^{2}}{M_{2}^{(j)}}+2\left[a_{2}{ }^{(j)}\right]^{2}\left[M_{2}{ }^{(j)}\right]\right\}+\frac{1}{4 \sqrt{\pi}} \frac{\left[M_{0}{ }^{(j)}\right]^{5 / 2}}{\left[M_{2}{ }^{(j)}\right]^{1 / 2}} \\
& (j=1,2), \\
& H_{\text {int }}=2 \kappa\left\{\frac{2\left[M_{0}{ }^{(1)} M_{0}{ }^{(2)}\right]^{3 / 2}\left[M_{2}^{(1)} M_{2}^{(2)}\right]^{1 / 2}}{M}\right\}^{1 / 2} \\
& \times \operatorname{Re}\left(\frac{\exp \left\{i\left[a_{0}{ }^{(2)}-a_{0}^{(1)}\right]\right\}}{\sqrt{(1-i d)}}\right)+\frac{\gamma}{\sqrt{2 \pi}}\left\{\frac{\left[M_{0}^{(1)} M_{0}^{(2)}\right]^{3}}{M}\right\}^{1 / 2},
\end{aligned}
$$


$\left.a_{2}^{(1)}\right] M_{2}{ }^{(1)} M_{2}{ }^{(2)} / M$. The evolution equations for the momenta and their conjugate variables are readily obtained from the total Hamiltonian $H$ in the usual way [see Eqs. (34)]. Integration of these equations for different choices of the initial conditions permits a study of the dynamical behavior of soliton parameters along the fiber. We have not been able to find additional invariants, besides the trivial ones associated with the conservation of energy, $E=M_{0}^{(1)}+$ $M_{0}{ }^{(2)}$, and with the conservation of momentum. ${ }^{47}$ Therefore a numerical integration of the trajectories of the dynamical system that is described by Eqs. (34)-(36) was necessary.

We shall discuss first the performance of the finite-dimensional representation in the simulation of ultrashort-pulse or soliton-switching experiments. ${ }^{37-41}$ A typical situation involves the study of the transmission characteristics of a coupler when one is varying the peak power of a pulse of given temporal width that is initially launched into one of the two modes. Beam-propagation simulations show that the pulses may switch between the two modes at the output without appreciable distortion in their shape, when the input peak power of the pulses grows larger than a certain critical switching power. ${ }^{46,47}$

A fundamental soliton of a single NLS equation is represented, in the spirit of our approach, by Eq. (A1), with $M_{0}{ }^{(i)} \sigma_{j}$ $=(\pi)^{1 / 2}$. Here we set the normalized input variance $\sigma_{j}^{2}=$ $(2)^{-1 / 2}$, so that the fundamental soliton corresponds to a normalized amplitude equal to 1 [i.e., $u_{0}=1$ in Eq. (A1)]. In the following calculations, the peak power $p$ of the pulses is normalized by the continuous-wave critical power $P_{c}: p=$ $P_{p} / P_{c}=u_{0}^{2}|1-\gamma| / 4 \kappa=\left[M_{0}^{(1)}+M_{0}^{(2)}\right]|1-\gamma| / 4 \kappa(2)^{1 / 4}(\pi)^{1 / 2}$.

In Figs. 1-4 we present a comparison of calculations obtained by integration of the representative system of ODE's (dashed curves) or by numerical integration of the PDE's by the beam-propagation method (solid curves). These calculations involve the evolution of typical parameters that characterize the pulses along a dual-core fiber (see Subsection 2.C): Figs. 1-4 show the pulse energy (which is equal to $M_{0}$ ) and the pulse width (i.e., $\sigma$ ) versus the normalized propagation distance. In Figs. 1 and 2 we simulate launching into one core of the fiber coupler a fundamental soliton with peak power equal to the critical continuous-wave power (i.e., $k=$ 0.25 and $p=1$ ), whereas Figs. 3 and 4 show the case in which $\kappa=0.25$ and $p=1.5$. Here the peak power of the input pulse is larger than the fundamental soliton power but still lower than that of the first higher-order (i.e., spatially periodic) soliton. Note that the propagation distance is given in units of the soliton characteristic length: one coupling length corresponds in Figs. $1-4$ to $z=2 \pi$. Whenever the peak power is lower than a certain critical power, complete and periodic energy exchange occurs (see Fig. 1). On the other hand, for powers larger than the above value of power only a small fraction of the total energy is coupled back and forth between the modes (see Fig. 3). As is clear by inspecting Figs. 1-3, almost complete switching of the output energy may be obtained in a one-coupling-length long dual-core nonlinear directional coupler (i.e., for $z=2 \pi$ ). This may be seen as a consequence of the halving of the period of the energy exchange that occurs when the input peak power grows from $P=P_{c}=P_{s}$ to $P=1.5 P_{c}$. Figures 1 and 3 show that a remarkable agreement exists between the finite-dimensional representation and the beam-propagation calculations, as far as the energy exchange is concerned, over relatively long propagation distances.
Consider next the pulse compression that may result from studying the evolution of pulse widths along the coupler. Figure 2 illustrates the case of a pulse that is launched at the input with $p=1$. Substantial temporal broadening may occur in a pulse that travels in the input mode whenever energy is transfered into the other mode: this is because, when the peak power grows smaller, the nonlinearity cannot balance dispersive broadening any longer. For the same reason, pulse compression occurs as the energy is coupled back. Meanwhile, the width of the pulse that propagates in the coupled mode grows only slightly larger over the same distance. Note the discrepancy that occurs between the predictions of the present method (dashed curves) and beam-propagation simulations (solid curves) in the estimate of the degree of temporal broadening of the pulse that propagates in the input mode. The situation is rather different

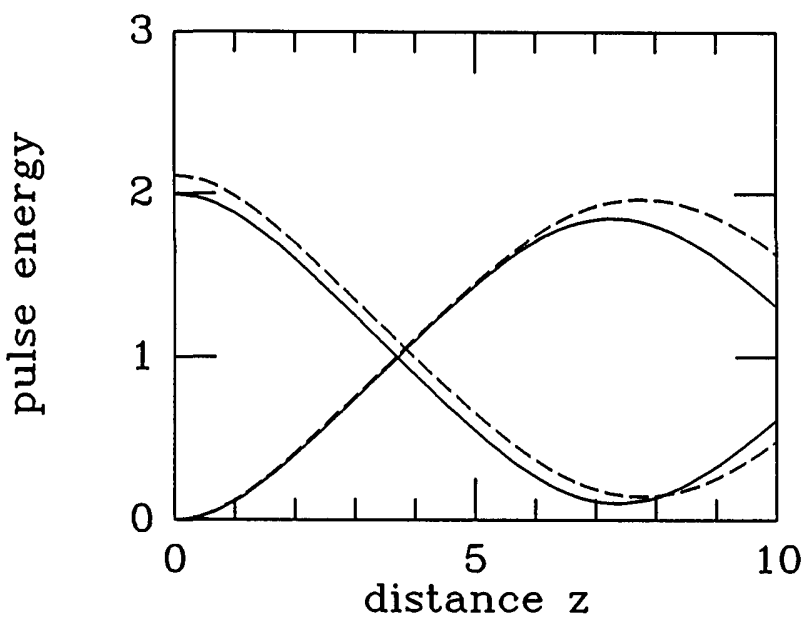

Fig. 1. Evolutions of the pulse energies along a dual-core nonlinear directional coupler ( $z$ is in units of soliton length $z_{c}$ ) when a fundamental soliton $(\kappa=0.25, p=1)$ is launched at the input of one guide. The dashed curve is obtained by integration of the ODE's, while the solid curve is obtained by numerical integration with the beampropagation method of the PDE's.

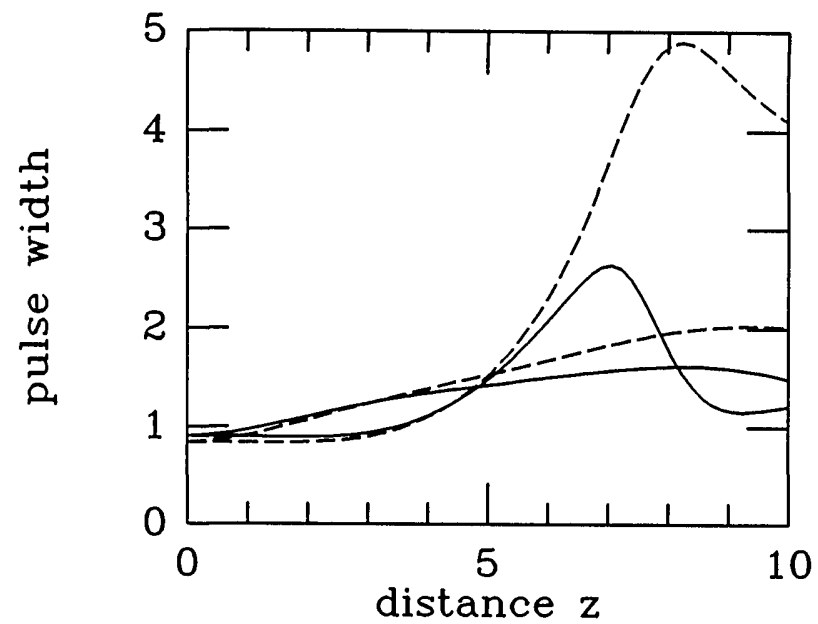

Fig. 2. Evolution of the pulse widths (standard deviations $\sigma_{\mathfrak{i}}, i=1$; 2) along a dual-core nonlinear directional coupler when a fundamental soliton $(k=0.25, p=1)$ is launched at the input of one channel. The dashed curve is obtained by integration of the ODE's, while the solid curve is obtained by numerical integration by the beam-propagation method of the PDE's. 


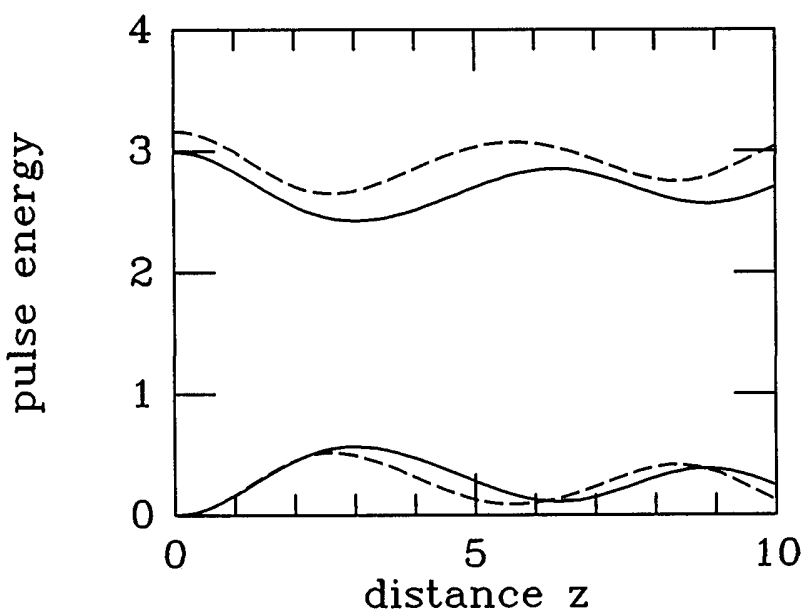

Fig. 3. Same as in Fig. 1 but with $p=1.5$.

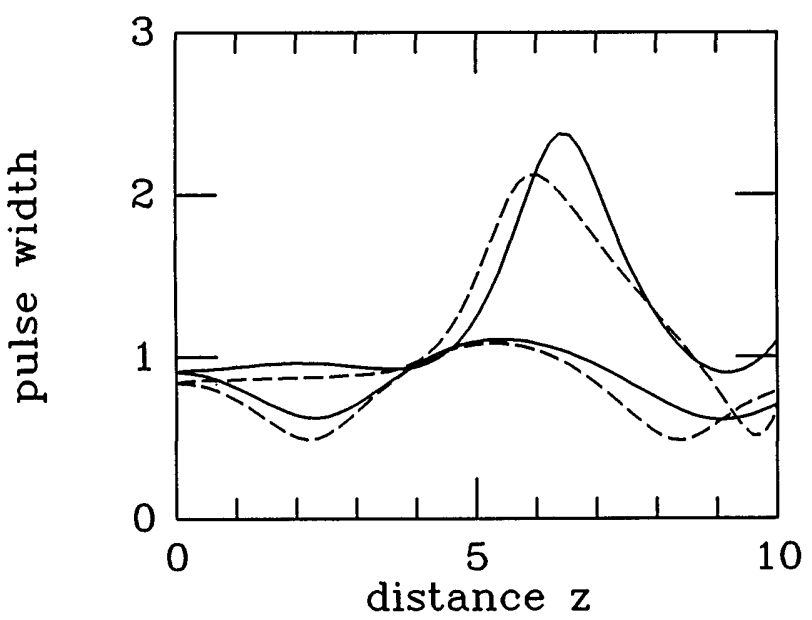

Fig. 4. Same as in Fig. 2 but with $p=1.5$.

when the input power is raised to the value $p=1.5$. Stable oscillations in the broadening and compression of the input pulse width occur with a spatial period that is the same as that of the energy transfer: the minima in the width correspond to the minima in the energy that remains in the pulse. At the same time, the amplitude of the oscillations in the compression of the coupled pulse width gets substantially larger with the distance. In this case (see Fig. 4) the agreement between the results of the two methods is satisfactory. Note that the small discrepancy in Figs. 1-4 between the initial values of the energies and the widths for the solid and dashed curves is due to the fact that the beam-propagation method simulations were carried out by using input pulses with hyperbolic secant (instead of Gaussian) profiles.

In Fig. 5 we compare three straight-through nonlinear energy transmissions \{defined as $M_{0}{ }^{(1)}\left(z=L_{c}\right) /\left[M_{0}{ }^{(1)}+\right.$ $M_{0}{ }^{(2)}$, where $i=1$ or 2 indicates the mode\} from a onecoupling-length-long dual-core-fiber nonlinear directional coupler. The switching curves were obtained by numerical integration of Eqs. (34)-(36) with input pulses of the form of Eq. (A1) (solid curve), by the beam-propagation method solution of Eqs. (18) (dotted-dashed curve, where squares represent actual calculated points), and by solving the coupled-mode equations in the continuous-wave limit (dashed curve). In solving Eqs. (34)-(36) we took $\gamma=0$ and $\kappa=0.25$ :
As can be seen, with this choice of parameters (which yields $P_{c}=P_{s}$ ), good agreement exists, both in the shape of the characteristics and in the value of the switching power, between the description of Eqs. (17) and (18) and that of Eqs. (34)-(36). Note that in the pulsed case the effective switching power, as estimated from energy-transmission curves, is shifted toward higher powers with respect to the continuouswave case. A qualitative explanation of this phenomenon is given in Appendix B. We show there that, based on the present model and under reasonable approximations, the evolution of the energy of two coupled solitons may be well approximated by the evolution of two equivalent continuous waves, with an effective power that, as one would expect, turns out to be smaller than the peak power of the input pulse.

Figure 6 shows energy transmissions [calculated by means of Eqs. (34)-(36)] from a dual-core coupler as a function of input pulse peak power for three different values of the ratio $P_{c} / P_{s}$. A reduction of the normalized coupling $\kappa$ leads to

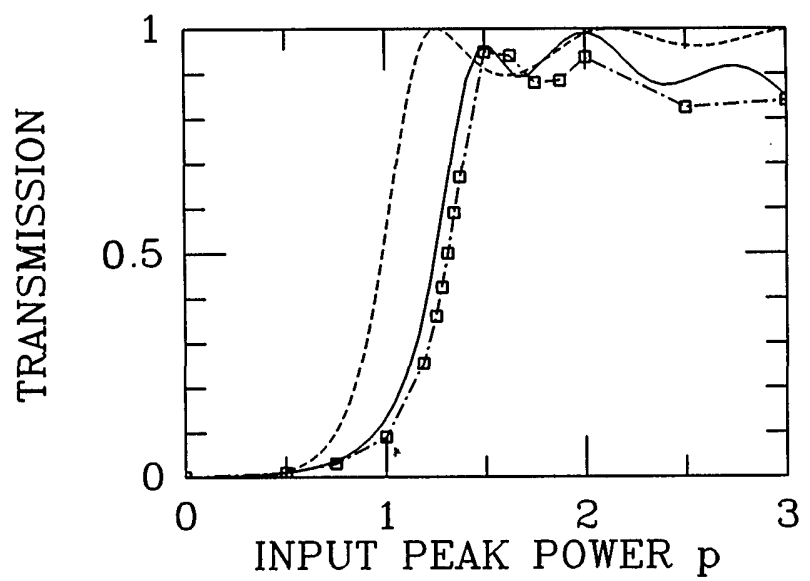

Fig. 5. Nonlinear switching characteristics for a one-couplinglength-long dual-core nonlinear directional coupler fiber. The dashed curve shows stationary power transmissivity versus power, while the other two curves show energy transmissions that are obtained either with the beam-propagation method integration of the PDE's (dotted-dashed curve with squares) or by integration of the present ODE's (solid curve), with $\kappa=1 / 4$ and $\gamma=0$.

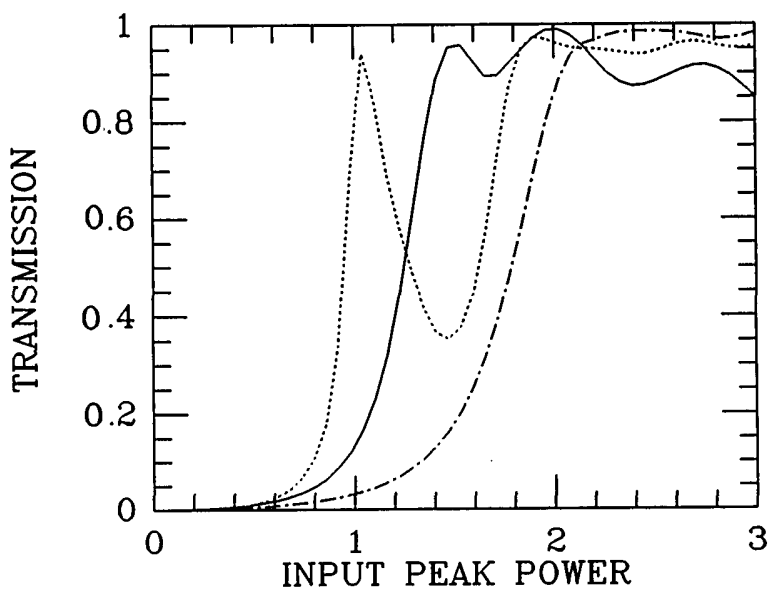

Fig. 6. Average transmissions versus pulse peak power for different values of the linear coupling $\kappa$, for a $L_{c}$-long dual-core nonlinear directional coupler: $\kappa=1 / 8$ (dotted-dashed curve), $k=1 / 4$ (solid curve), $\kappa=1$ (dotted curve). 
larger effective switching powers and smoother switching characteristics. In particular, when $\kappa=1 / 8$ the switching curve is close to the continuous-wave case with an effective switching power of $p=2$. However, for values of $k$ as high as 1 , the switching characteristic departs strongly from the stationary case. Values of $k$ larger than $1 / 4$ correspond to a situation in which, at the critical power $p=1$, pulses with peak power larger than the fundamental soliton power or even higher-order solitons (for $\kappa=n / 4, n>1$, integer) are launched at the input. Note that increasing $\kappa$ while keeping the normalized input pulse width fixed is equivalent to reducing the input pulse width with a given value of the coupling distance.

Energy transmissions computed over longer propagation distances and with relatively high values of $K$ may display a complex behavior. For example, Fig. 7 shows the transmission computed for $\kappa=1$ and $\gamma=0$ and for a coupler of length $L=2 L_{c}$. In contrast with the regular (oscillatory with power) switching characteristics that pertain to the stationary case, irregular soliton transmission curves are obtained because of a stochastic behavior of the spatial evolution of the parameters associated with the interacting pulses. The history of pulse energy versus length for different input conditions is displayed in Figs. 8 and 9. In Fig. 8 we show the energy $M_{0}{ }^{(1)}$ of a circularly polarized pulse that propagates in a birefringent silica fiber (i.e., $\gamma=2$ and $B=1 / 3$, with $\kappa=$ 1) over a relatively long distance (20 characteristic soliton units). Figure 9 shows evolutions of circular polarization components of a pulse that propagates in a birefringent fiber, again with $\kappa=1$. The initial polarization is close to either the slow (dashed curve) or fast (solid curve) birefringence axis (this is spatially unstable in the continuous-wave regime $^{32,33}$ ). Spatially disordered evolution may result for the pulse parameters described by the model of Eqs. (34)(36) when the initial polarization of the pulse is close to a spatially unstable eigenstate.

The disordered evolution of the pulse energies that is revealed by the trajectories and the switching characteristics is associated with the nonintegrability of the Hamiltonian dynamical system of Eqs. (34)-(36), which may lead to chaos. However, owing to the high number of degrees of freedom of the system, a more detailed numerical or analytical

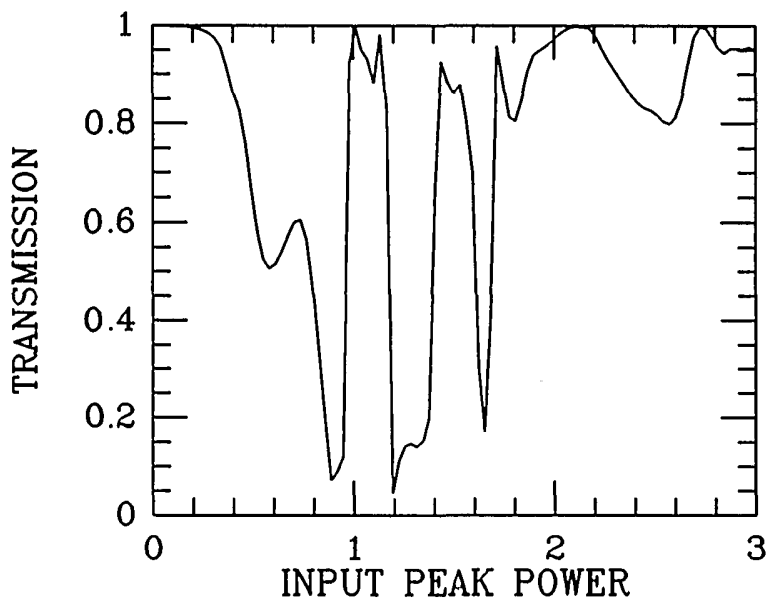

Fig. 7. Average transmission for a dual-core nonlinear directional coupler of length $2 L_{c}$ and $\kappa=1$.

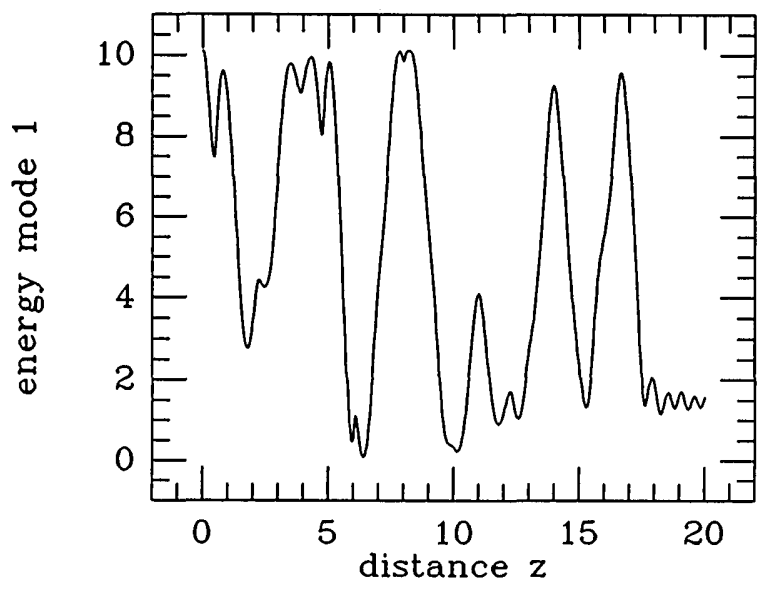

Fig. 8. Irregular spatial evolution of the total energy contained in the initially excited right-handed circular polarization component of a pulse traveling in a birefringent fiber, for $\kappa=1$. $z$ is in units of soliton characteristic length.

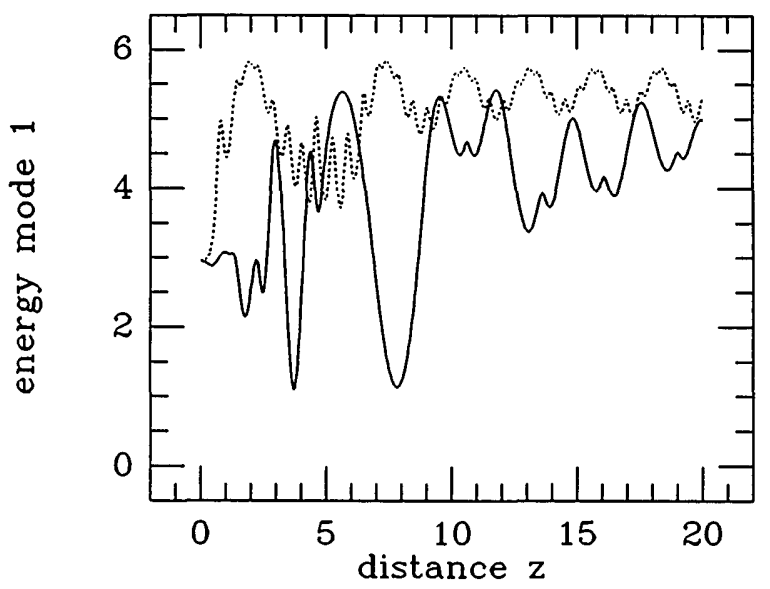

Fig. 9. Same as Fig. 8, when the input pulse is polarized close to the slow (dotted curve) or fast (solid curve) axis of the fiber.

study of the irregular behavior and of the chaoticity domains is beyond the scope of this work. We wish, however, to point out that, in order to assess the physical significance of the spatially disordered evolutions that are exhibited by the time-independent parameters in the framework of the present finite-dimensional representation, one should examine the effects of increasing the accuracy of the representation, for example, by means of adding new parameters or by direct simulation of the solutions of the full system of PDE's.

\section{CONCLUSIONS}

We have presented a general method for dealing with nonlinear pulse propagation of coupled modes in guiding structures. It allowed us to reduce the system of nonlinear coupled PDE's, which describe time-dependent propagation, to a set of ODE's for a finite number of parameters, characterizing the phases and the intensities of modal amplitudes. Starting from the field Lagrangian, we have demonstrated that these two sets of parameters are conjugate variables through a suitable Hamiltonian, which is readily evaluated, 
once the shape of the pulses and the number of parameters have been chosen, by performing simple integrals.

By applying the method to the investigation of soliton switching in two-mode fiber couplers, we have been able to reproduce the characteristic features of the energy transfer and temporal compression of the interacting pulses, in good agreement with results that are obtainable by numerically integrating the system of coupled NLS equations. Extensions of the present method may be useful for dealing with different systems of coupled PDE's, representing physical interactions that are also outside the field of optics.

\section{APPENDIX A}

In the following example we explicitly calculate the Hamiltonian for the case $N=2$; in other words, we assume that each pulse can be simply characterized by three momenta $\left(M_{0}, M_{1}, M_{2}\right)$ associated with the pulse energy, the pulse walk-off from the center-of-mass, and the pulse width, respectively. We further assume a Gaussian shape for the pulses, so that

$\left|u_{j}(z, t)\right|^{2}=u_{j 0}^{2} \exp \left[-\frac{\left(t-\tau_{j}\right)^{2}}{2 \sigma_{j}^{2}}\right]=\frac{M_{0}{ }^{(j)}}{\sqrt{2 \pi} \sigma_{j}} \exp \left[-\frac{\left(t-\tau_{j}\right)^{2}}{2 \sigma_{j}^{2}}\right]$

where

$$
\tau_{j}=\frac{M_{1}^{(j)}}{M_{0}^{(j)}}, \quad \sigma_{j}^{2}=\frac{M_{2}^{(j)}}{M_{0}^{(j)}}-\tau_{j}^{2} .
$$

The total Hamiltonian may be written as a sum of two parts, which describe self-interaction and the mutual interaction between the two pulses, respectively. By evaluating all the integrals that appear in Eq. (30), one finds that

\section{APPENDIX B}

Here we show how the switching behavior of interacting solitons in fiber couplers can be explained, in a qualitative way, by means of a simple transformation of the Hamiltonian calculated with the method reported in this paper [see Eqs. (35) and (36)]. More precisely, we show that, under certain assumptions, the evolution equations that govern the pulse propagation in the framework of our model are equivalent to the coupled-mode equations that describe the propagation of continuous waves in a time-stationary regime.

In particular, we consider here the evolution of two solitonlike unmodulated pulses [i.e., $a_{j}^{(2)}=0$ ], which evolve with constant and equal variance. This assumption, though it may appear arbitrary, turns out to be well supported by numerical simulations, performed either by integrating the equations obtained by means of the present model or by the beam-propagation method. In fact, by investigating the behavior of pulses that are launched with fixed width and increasing peak power, as is done when one is computing the energy transmissions, it turns out that, for not-too-large $\kappa$ 's, they exhibit only small periodic variations of their widths. Even at powers lower than the soliton power, they couple without experiencing substantial width variations (see Figs. 2-4), at least over the lengths considered. According to these considerations, the Hamiltonian given in Eq. (36), written as a function of the pulse widths $\sigma_{1}=\sigma_{2} \equiv \sigma=\left(M_{2} /\right.$ $\left.M_{0}\right)^{1 / 2}$, takes the form

$$
\begin{aligned}
H & =H\left[M_{0}{ }^{(i)}, a_{0}{ }^{(i)}\right]=\frac{\left[M_{0}{ }^{(1)}\right]^{2}+\left[M_{0}{ }^{(2)}\right]^{2}}{4 \sqrt{\pi} \sigma} \\
& +2 \kappa\left[M_{0}{ }^{(1)} M_{0}{ }^{(2)}\right]^{1 / 2} \cos \left[a_{0}{ }^{(1)}-a_{0}{ }^{(2)}\right]+\gamma \frac{M_{0}{ }^{(1)} M_{0}{ }^{(2)}}{2 \sqrt{\pi} \sigma},
\end{aligned}
$$

$$
\begin{aligned}
& H=\sum_{j=1,2} H_{j}+H_{\mathrm{int}}=\sum_{j=1,2}\left(\left\{\frac{m}{V}(-1)^{j}\left[a_{1}{ }^{(j)} M_{0}{ }^{(j)}+2 a_{2}{ }^{(j)} M_{1}{ }^{(j)}\right]\right\}-\frac{n}{V} \operatorname{Im}\left[\left[\frac{M_{0}^{(1)} M_{0}^{(2)}}{2\left(\sigma_{1}{ }^{2}+{\sigma_{2}}^{2}\right)}\right]^{1 / 2} \frac{i a_{1}{ }^{(3-j)}}{\sqrt{1-i d_{j}}} \exp \left\{i\left(a_{0}{ }^{(3-j)}-a_{0}{ }^{(j)}\right]\right\}\right.\right. \\
& \left.\times \exp \left(-\frac{\tau_{1}^{2}}{4{\sigma_{1}}^{2}}-\frac{\tau_{2}^{2}}{4{\sigma_{2}}^{2}}+\frac{\left\{\frac{\tau_{1}}{2{\sigma_{1}}^{2}}+\frac{\tau_{2}}{2{\sigma_{2}}^{2}}+i\left[a_{1}^{(3-j)}-a_{1}^{(j)}\right]\right\}^{2}}{1-i d} \sigma^{2}\right)\right] \\
& \left.-\mp \frac{1}{2}\left\{\frac{M_{0}^{(j)}}{4 \sigma_{j}^{2}}+\left[a_{1}^{(j)}\right]^{2} M_{0}^{(j)}+4\left[a_{2}^{(j)}\right]^{2} M_{2}{ }^{(j)}\right\}-\Delta(-1)^{j} M_{0}^{(j)}+\frac{\left[M_{0}^{(j)}\right]^{2}}{4 \sqrt{\pi} \sigma_{j}}\right) \\
& +2 \kappa \operatorname{Re}\left[\left[\frac{M_{0}^{(1)} M_{0}^{(2)}}{1-i d}\left(\frac{2 \sigma_{1} \sigma_{2}}{\sigma_{1}{ }^{2}+\sigma_{2}{ }^{2}}\right)\right]^{1 / 2} \exp \left\{i\left[a_{0}{ }^{(2)}-a_{0}{ }^{(1)}\right]\right\} \exp \left(-\frac{\tau_{1}{ }^{2}}{4{\sigma_{1}}^{2}}-\frac{\tau_{2}{ }^{2}}{4{\sigma_{2}}^{2}}+\frac{\left\{\frac{\tau_{1}}{2 \sigma_{1}{ }^{2}}+\frac{\tau_{2}}{2 \sigma_{2}{ }^{2}}-i\left[a_{1}{ }^{(2)}-a_{1}{ }^{(1)}\right]\right\}^{2}}{1-i d} \sigma^{2}\right)\right] \\
& +\gamma \frac{M_{0}^{(1)} M_{0}^{(2)}}{\sqrt{2 \pi}} \frac{1}{\left(\sigma_{1}^{2}+\sigma_{2}^{2}\right)^{1 / 2}} \exp \left[-2 \sigma^{2}\left(\frac{\tau_{1}}{\sigma_{1}^{2}}+\frac{\tau_{2}}{\sigma_{2}^{2}}\right)^{2}\right] \\
& +\rho \operatorname{Re}\left[\frac{M_{0}^{(1)} M_{0}^{(2)}}{\left[2 \pi\left(\sigma_{1}{ }^{2}+\sigma_{2}{ }^{2}\right)(1-i d)\right]^{1 / 2}} \exp \left\{i\left[a_{0}{ }^{(2)}-a_{0}{ }^{(1)}\right]\right\} \exp \left(-\frac{\tau_{1}{ }^{2}}{2 \sigma_{1}{ }^{2}}-\frac{\tau_{2}{ }^{2}}{2{\sigma_{2}}^{2}}+\frac{\left\{\frac{\tau_{1}}{\sigma_{1}{ }^{2}}+\frac{\tau_{2}}{\sigma_{2}{ }^{2}}+i 2\left[a_{1}^{(2)}-a_{1}^{(1)}\right]\right\}^{2}}{1-i d} \frac{\sigma^{2}}{2}\right]^{2},\right.
\end{aligned}
$$

where $\sigma^{2}=\left(1 / \sigma_{1}{ }^{2}+1 / \sigma_{2}{ }^{2}\right)^{-1}, d=4 \sigma^{2}\left[a_{2}{ }^{(2)}-a_{1}^{(2)}\right]$, and $d_{j}=$ $4 \sigma_{j}^{2}\left[a_{2}^{(2)}-a_{1}^{(2)}\right]$. where we have dropped the dispersive term since it is $z$ invariant for constant $\sigma$. From Eq. (A1) it is immediately 
verified that the variables $y_{i}=M_{0}^{(i)} /\left[M_{0}^{(1)}+M_{0}^{(2)}\right]$ are conjugate to the $a_{i}=a_{0}{ }^{(i)}$ through the Hamiltonian

$$
H^{\prime}\left(y_{i}, a_{i}\right)=\frac{1}{M_{0}^{(1)}+M_{0}^{(2)}} H\left[M_{0}^{(i)}, a_{i}\right] .
$$

Since the Hamiltonian in Eq. (B2) depends only on the phase difference $a=a_{1}-a_{2}$, and the total energy is conserved (i.e., $y_{1}+y_{2}=1$ ), the relevant variables turn out to be the phase and the energy difference, $a$ and $y=y_{1}-y_{2}$, respectively. It is easy to show, with a canonical transformation, that the variables $(y, a)$ are conjugate variables, with

$$
\begin{gathered}
H=H(y, a)=p_{1} y^{2}+\sqrt{1-y^{2}} \cos (a), \\
\frac{\mathrm{d} a}{\mathrm{~d} \zeta}=\frac{\partial H}{\partial y}, \quad \frac{\mathrm{d} y}{\mathrm{~d} \zeta}=-\frac{\partial H}{\partial a},
\end{gathered}
$$

where we have introduced the propagation distance in units of beat length $\zeta=2 \kappa z=2 \pi z_{1} / L_{b}, z_{1}$ being the propagation distance in real units, and the normalized peak power $p_{1}=p /$ $(2)^{1 / 2}$. This Hamiltonian is formally equivalent to the integrable Hamiltonian that governs the nonlinear evolution of two coupled modes in a fiber coupler in the stationary regime. ${ }^{55,56}$ In the continuous-wave case, Eq. (B3) holds, provided that $p_{1}$ is given the meaning of total continuous-wave power normalized to the critical one, say, $p_{\mathrm{cw}}=P / P_{c}$, the variables $a$ and $y$ being, respectively, the phase and continuous-wave power (normalized to the total power, that is, $y \in$ $[-1,1])$ differences.

Such formal equivalence implies that, under our assumptions, the dynamical evolution of the interacting solitonlike pulses follows the corresponding evolution of the continuous waves, where the role played by the power of the waves is now played by the energy (i.e., the time integral of the power) of the pulses. Furthermore, the continuous-wave normalized power $p_{\mathrm{cw}}$ is replaced, for solitonlike pulses, by the power $p_{1}=p /(2)^{1 / 2}$, where $p$ is the input peak power of the pulse divided by the continuous-wave critical power $P_{c}$. Since switching for continuous waves is expected, on the basis of Eq. (B3) at the critical power, ${ }^{55}$ that is, for $p_{\mathrm{cw}}=1$ (see the dashed curve in Fig. 5), this qualitatively explains why switching of the pulse energy, which would be expected for $p_{1}=1$, actually occurs for larger values of power $p$ (see Fig. 5).

\section{ACKNOWLEDGMENTS}

We are grateful to F. Gori and G. De Biase for making available to us the computing facilities of the Dipartimento di Fisica and of the Istituto di Astronomia of the University of Rome, "La Sapienza." This research was carried out under an agreement between the Fondazione Ugo Bordoni and the Istituto Superiore Poste e Telecomunicazioni.

\section{REFERENCES}

1. B. Crosignani, C. H. Papas, and P. Di Porto, Opt. Lett. 6, 61 (1981); B. Crosignani, A. Cutolo, and P. Di Porto, J. Opt. Soc. Am. 72, 1136 (1982); see also S. Solimeno, B. Crosignani, and P. Di Porto, Guiding, Diffraction and Confinement of Optical Radiation (Academic, New York, 1986), Chap. VIII.

2. V. E. Zakharov, Zh. Eksp. Teor. Fiz, 53, 1733 (1967) [Sov. Phys. JETP 26, 994 (1968)].

3. V. I. Karpman, Nonlinear Waves in Dispersive Media (Novosibirsk State University, Novosibirsk, 1968).
4. V. E. Zakharov and A. B. Shabat, Zh. Eksp. Teor. Fiz. 61, 118 (1971) [Sov. Phys. JETP 34, 62 (1972)].

5. V. E. Zakharov and A. B. Shabat, Zh. Eksp. Teor. Fiz. 64, 1627 (1973) [Sov. Phys. JETP 37, 823 (1973)].

6. A. Hasegawa and F. Tappert, Appl. Phys. Lett. 23, 142 (1973).

7. A. Hasegawa and F. Tappert, Appl. Phys. Lett. 23, 171 (1973).

8. For a review on correcting terms in the NLS equation when dealing with femtosecond pulses, see, for example, Y. Kodama and A. Hasegawa, IEEE J. Quantum Electron. QE-23, 510 (1987).

9. L. F. Mollenauer, R. H. Stolen, and J. P. Gordon, Phys. Rev. Lett. 45, 1095 (1980).

10. D. Krokel, N. J. Halas, G. Giuliani, and D. Grischkowsky, Phys. Rev. Lett. 60, 29 (1988); A. M. Weiner, J. P. Heritage, R. J. Hawkins, R. N. Thurston, E. M. Kirschner, D. E. Leaird, and W. J. Tomlinson, Phys. Rev. Lett. 61, 2445 (1988).

11. S. V. Manakov, Zh. Eksp. Teor. Fiz. 65, 505 (1973) [Sov. Phys. JETP 38, 248 (1974)].

12. V. E. Zakharov and E. I. Schulman, Physica 4D, 270 (1982); R. Sahadevan K. M. Tamizhmani, and M. Lakshmanan, J. Phys. A 19, 1783 (1986).

13. R. Hirota, "Direct methods in soliton theory," in Solitons, R. K. Bullough and P. J. Caudry, eds. (Springer-Verlag, Berlin, 1980), Chap. 5 .

14. A. L. Berkhoer and V. E. Zakharov, Sov. Phys. JETP 31, 486 (1970).

15. C. R. Menyuk, IEEE J. Quantum Electron. QE-23, 174 (1987); Opt. Lett. 12, 614 (1988); J. Opt. Soc. Am. B 5, 392 (1988).

16. D. N. Christodoulides and R. I. Joseph, Opt. Lett. 13, 53 (1988); M. V. Tratnik and J. E. Sipe, Phys. Rev. A 38, 2011 (1988).

17. S. Trillo, S. Wabnitz, E. M. Wright, and G. I. Stegeman, Opt. Lett. 13, 871 (1988); V. V. Afanas'ev, E. M. Dianov, A. M. Prokhorov, and V. N. Serkin, Pis'ma Zh. Eksp. Teor. Fiz. 48, 588 (1988) [JE'TP Lett. 48, 638 (1988)].

18. D. N. Christodoulides, Phys. Lett. A 132, 451 (1988).

19. D. J. Kaup and A. C. Newell, Proc. R. Soc. London Ser. A 361, 413 (1978).

20. A. Bondeson, D. Anderson, and M. Lisak, Phys. Sci. 20, 479 (1979).

21. D. Anderson, Phys. Rev. A 27, 3135 (1983).

22. D. Anderson and M. Lisak, Phys. Rev. A 32, 2270 (1985); Opt. Lett. 11, 174 (1986).

23. V. I. Karpman and V. V. Solov'ev, Physica D 3, 487 (1981).

24. E. Caglioti, B. Crosignani, and P. Di Porto, Phys. Rev. A 38, 4036 (1988).

25. S. Wiggins, Global Bifurcations and Chaos-Analytical Methods (Springer-Verlag, Berlin, 1988).

26. R. A. Fisher and W. K. Bishel, J. Appl. Phys. 46, 4921 (1975); see also D. Yevick and B. Hermansson, Opt. Commun. 47, 101 (1983).

27. B. Daino, S. Trillo, S. Wabnitz, and G. I. Stegeman, Proc. Soc. Photo-Opt. Eng. 836, 254 (1987); S. Wabnitz and S. Trillo, Digest of the Topical Meeting on Nonlinear Guided-Wave Phenomena: Physics and Applications (Optical Society of America, Washington, D.C., 1989), pp. 198-201.

28. S. M. Jensen, IEEE J. Quantum Electron. QE-18, 1580 (1982).

29. A. A. Maier, Sov. J. Quantum Electron. 12, 1490, 1982; 14, 101 (1984); 16, 892 (1986).

30. B. Daino, G. Gregori, and S. Wabnitz, J. Appl. Phys. 58, 4512 (1985).

31. S. Wabnitz, E. M. Wright, C. T. Seaton, and G. I. Stegeman, Appl. Phys. Lett. 49, 838 (1986); S. Trillo and S. Wabnitz, Appl. Phys. Lett. 49, 752 (1986); J. Opt. Soc. Am. B 5, 483 (1988).

32. B. Daino, G. Gregori, and S. Wabnitz, Opt. Lett. 11, 42 (1986); F. Matera and S. Wabnitz, Opt. Lett. 11, 467 (1986).

33. H. G. Winful, Opt. Lett. 11, 33 (1986).

34. A. Mecozzi, S. Trillo, S. Wabnitz, and B. Daino, Opt. Lett. 12 , 275 (1987); for the linear characteristics of this fiber, see also, $R$. H. Stolen, A. Ashkin, W. Pleibel, and J. M. Dziedzic, Opt. Lett. 9, 300 (1984).

35. S. Trillo, S. Wabnitz, and G. I. Stegeman, IEEE J. Lightwave Technol. 6, 971 (1988).

36. Y. Silberberg and G. I. Stegeman, Appl. Phys. Lett. 50, 801 (1987).

37. S. Trillo, S. Wabnitz, R. H. Stolen, G. Assanto, C. T. Seaton, and G. I. Stegeman, Appl. Phys. Lett. 49, 1224 (1986). 
38. D. D. Gusovskii, E. M. Dianov, A. A. Maier, V. B. Neustreuev, E. I. Shklovskii, and A. Shcherbakov, Sov. J. Quantum Electron. 15, 1523 (1985); D. D. Gusovskii, E. M. Dianov, A. A. Maier, V. B. Neustreuev, V. V. Osiko, A. M. Prokhorov, K. Yu. Sitarkii, and A. Shcherbakov, Sov. J. Quantum Electron. 17, 724 (1985); A. A. Maier, Yu. N. Serdyuchenko, K. Yu. Sitarskii, M. Ya. Shchelev, and I. A. Shcherbakov, Sov. J. Quantum Electron. 17, 735 (1987).

39. S. R. Friberg, Y. Silberberg, M. K. Oliver, M. J. Andrejco, M. A. Saifi, and P. W. Smith, Appl. Phys. Lett. 51, 1135 (1987); S. R. Friberg, A. M. Weiner, Y. Silberberg, B. G. Sfez, and P. W. Smith, Opt. Lett. 13, 904 (1988).

40. S. Trillo, S. Wabnitz, N. Finlayson, W. C. Banyai, C. T. Seaton, G. I. Stegeman, and R. H. Stolen, Appl. Phys. Lett. 53, 837 (1988); S. Trillo, S. Wabnitz, W. C. Banyai, N. Finlayson, C. T. Seaton, G. I. Stegeman, and R. H. Stolen, IEEE J. Quantum Electron. 25, 104 (1989).

41. A. M. Weiner, Y. Silberberg, H. Fouckhardt, D. E. Leaird, M. A. Saifi, M. J. Andrejco, and P. W. Smith, in Digest of Conference on Lasers and Electro-Optics (Optical Society of America, Washington, D.C., 1989), pp. 226-227.

42. A. M. Weiner and J. P. Heritage, Rev. Phys. Appl. 22, 1619 (1987); A. M. Weiner, J. P. Heritage, and J. A. Salehi, Opt. Lett. 13, 300 (1988).

43. N. J. Doran and D. Wood, J. Opt. Soc. Am. B 4, 1843 (1987); Opt. Lett. 13, 56 (1988); N. J. Doran, K. J. Blow, and D. Wood, Proc. Soc. Photo-Opt. Instrum. Eng. 836, 238 (1987).
44. K. J. Blow, N. J. Doran, and B. K. Nayar, Opt. Lett. 14, 754 (1989).

45. K. J. Blow, N. J. Doran, and D. Wood, Opt. Lett. 12, 202 (1987).

46. S. Trillo, S. Wabnitz, E. M. Wright, and G. I. Stegeman, Opt. Lett. 13, 672 (1988).

47. S. Trillo, S. Wabnitz, E. M. Wright, and G. I. Stegeman, Opt. Commun. 70, 166 (1989).

48. A. D. Boardman and G. S. Cooper, J. Opt. Soc. Am. B 5, 403 (1988).

49. F. Kh. Abdullaev, R. M. Abrarov, and S. A. Darmanyan, Opt. Lett. 14, 131 (1989).

50. E. M. Wright, G. I. Stegeman, and S. Wabnitz, Phys. Rev. A 40, 4455 (1989).

51. S. Trillo and S. Wabnitz, J. Opt. Soc. Am. B 6, 238 (1989).

52. P. D. Maker, R. W. Terhune, and C. M. Savage, Phys. Rev. Lett. 12, 507 (1964).

53. B. Crosignani and P. Di Porto, Opt. Acta 32, 2251 (1985).

54. S. Trillo, S. Wabnitz, and G. I. Stegeman, IEEE J. Quantum Electron. 25, 1907 (1989).

55. S. Wabnitz, Phys. Rev. Lett. 58, 1415 (1987); E. Caglioti, S. Trillo, and S. Wabnitz, Opt. Lett. 12, 1044 (1987); S. Trillo and S. Wabnitz, J. Opt. Soc. Am. B 5, 483 (1988).

56. E. Caglioti, S. Trillo, and S. Wabnitz, in Advances in Nonlinear Dynamics and Stochastic Processes II, G. Paladin and A. Vulpiani, eds. (World Scientific, Singapore, 1987), p. 225. 